\title{
Network Analysis of Posttraumatic Stress Experiences of Adults Seeking Psychological Treatment for Childhood Sexual Abuse
}

\author{
Orla McBride (iD , ${ }^{1}$ Philip Hyland (D) ${ }^{2}$ Jamie Murphy, ${ }^{1}$ and Ask Elklit ${ }^{3}$ \\ ${ }^{1}$ School of Psychology, Ulster University, Cromore Road, Coleraine, Co. Londonderry, Northern Ireland, BT52 1SA, United \\ Kingdom \\ ${ }^{2}$ Department of Psychology, Maynooth University, Maynooth, Co. Kildare, Ireland \\ ${ }^{3}$ National Centre for Psychotraumatology, Department of Psychology, University of Southern Denmark, Campusvej 55 Odense \\ M, DK-5230, Denmark
}

\begin{abstract}
Network analysis proposes that mental disorders may best be construed as causal systems embodied in networks of functionally interconnected symptoms. We employed network analysis to test how adult survivors of childhood sexual abuse (CSA) experienced symptoms of posttraumatic stress, using alternative conceptualizations of posttraumatic stress disorder (PTSD). Given the characteristics of the sample (i.e., the nature of and time since trauma), we hypothesized that (a) symptoms related to arousal would not be prominent in the networks and (b) symptoms related to negative alternations in cognition and mood (NACM) would be core components in the network. Danish adults seeking psychological treatment for CSA $(n=473)$ completed the Harvard Trauma Questionnaire and Trauma Symptom Checklist. Three alternative models (DSM-5, DSM-5 with dissociation, and ICD-11 complex PTSD [CPTSD]) were estimated using regularized partial correlation models. In the DSM-5 network, strong associations emerged for experiences of NACM (blame and guilt) and intrusions (thoughts and flashbacks). The addition of "depersonalization" and "derealization" to the DSM-5 model produced a strong association, but these experiences were largely unrelated to other PTSD clusters. In the CPTSD network, interpersonal problems and negative self-concept were central to the survivors' experiences. For this highly-specific survivor group who experienced traumatic CSA many years ago, experiences related to NACM appeared to be more central to the posttrauma experience than those of arousal. If replicated, these findings could help inform treatment plans for specific groups of survivors. Methodological implications as to the usefulness of network models in the psychopathological research literature are discussed.
\end{abstract}

The disease model of psychopathology, which proposes that "disorders," such as posttraumatic stress disorder (PTSD), are manifested by "symptoms," such as avoidance, hyperarousal, and recurrent nightmares (Borsboom, Cramer, Schmittmann, Epskamp, \& Waldorp, 2011), is inherently problematic. First, for this model to hold, it should be possible to separate conceptually the condition (e.g., PTSD) from its symptoms (e.g., hyperarousal). In the absence of robust evidence, such as key genetic markers or neural abnormalities, as to the root cause of mental disorders (Kendler, 2005), the main indicators of psychological distress are a list of "symptoms" outlined in psychiatric classification systems. It is not possible to be diagnosed with PTSD independent of symptoms

Correspondence concerning this article should be addressed to: Orla McBride, $\mathrm{PhD}$, School of Psychology, Ulster University, Cromore Road, Coleraine, Co. Londonderry, Northern Ireland, BT52 1SA United Kingdom. E-mail: o.mcbride@ulster.ac.uk

(c) 2019 International Society for Traumatic Stress Studies. View this article online at wileyonlinelibrary.com

DOI: $10.1002 /$ jts. 22381 that are intrusive or related to cognitive function, which are core symptoms for a diagnosis per the Diagnostic and Statistical Manual of Mental Disorders (DSM) or International Classification of Diseases (ICD). Second, in a latent variable modeling framework, the assumption of local independence must be satisfied; that is, observed variables are assumed to be statistically independent conditional on the latent variable (Borsboom, 2008). This assumption means that symptoms such as difficultly sleeping, hyperarousal, and recurrent nightmares co-occur within an individual only because they are all caused by a condition called PTSD and not because they are causally related. For many disorders, including PTSD, this seems to be an implausible assumption (Cramer et al., 2012).

If it is assumed that the associations between the observable components of psychological constructs such as PTSD are real (Cramer et al., 2012), then mental disorders may best be construed as causal systems embodied in networks of functionally interconnected symptoms (McNally et al., 2014). Network analysis, which conceptualizes symptoms as constitutive of the mental disorder rather than as a reflection of 
a latent entity (Robinaugh, LeBlanc, Vuletich, \& McNally, 2014), has gained attention in recent years (see Cramer et al., 2012).

McNally and colleagues (2014) generated a network model of PTSD using data from a sample of Chinese earthquake survivors who had a diagnosis of probable PTSD $(n=362$; $73 \%$ women). The results identified that (a) hypervigilance was central to the network, meaning it was a core feature of the survivors' experience; (b) "feelings that one's future will be cut short" acted as a bridge that connected both hypervigilance and intrusive memories with emotional numbness, and, in turn, with feelings of social disconnection and anhedonia; (c) there was bidirectionality between experiences; for example, hypervigilant survivors were prone to startle, which served to promote continued hypervigilance and thus increased the chronicity of the disorder; (d) irritability/anger was linked to sleep and concentration problems; and (e) intrusive thoughts, flashbacks, and nightmares were all connected, but physiological and emotion reactions to reminders of the trauma were not. Studies that have followed this pioneering work have produced varying results. For example, Sullivan, Smith, Lewis, and Jones (2016) found less support for the centrality of hypervigilance in a network of posttrauma experiences among university students who had witnessed an on-campus mass shooting but stronger evidence for the role of intrusive thoughts and anger. In a sample of U.S. veterans diagnosed with probable PTSD, Armour, Fried, Deserno, Tsai, and Pietrzak (2017) reported that experiences associated with guilt, shame, flashbacks, and reactivity to trauma reminders were central to the network.

Although recent work has indicated that the structures of PTSD networks across heterogeneous treatment-seeking samples have patterns of symptoms in common (Fried et al., 2018), it is plausible that the configuration of a posttrauma network may depend on the type of trauma that was experienced and duration of time since the trauma (Roberts, Gilman, Breslau, Breslau, \& Koenen, 2011). Much evidence has alluded to the role that arousal in the short-term aftermath of a violent trauma plays in increasing the likelihood for PTSD (Schell, Marshall, \& Jaycox, 2004). Symptoms from other clusters, mainly avoidance, tend to occur following periods of excessive arousal and reexperiencing (O'Donnell, Elliott, Lau, \& Creamer, 2007) in an attempt to establish cognitive equilibrium, which may be maladaptive and promote dysfunction (Clark \& Beck, 2011). Negative alterations in cognition and mood (NACM), including guilt, might be particularly prevalent among individuals who have been sexually abused (Kubany \& Manke, 1995), perhaps partly due to the personally invasive nature of this trauma type. In this study, we used network analysis to assess the PTSD experiences of adults seeking psychological treatment for childhood sexual abuse (CSA). We modeled alternative conceptualizations of PTSD as per the fifth edition of the $D S M(D S M-5)$ and complex PTSD (CPTSD) as per the 11th revision of the ICD (ICD-11), the latter of which acknowledges that disturbances in self-organization (DSO) are evident among individuals who have experienced chronic and repeated trauma (Cloitre, Garvert, Brewin, Bryant, \& Maercker, 2013), to test two hypotheses: (a) symptoms related to arousal would not be prominent in the networks and (b) symptoms of NACM would be central components of the networks.

\section{Method}

\section{Participants and Procedure}

Data were collected from adult attendees $(N=484)$ at four Danish treatment centers that provide psychological treatment for CSA victims. Further details on the study are available elsewhere (Elklit, Christiansen, Palic, Karsberg, \& Eriksen, 2014). Briefly, the treatment centers are supported by Denmark's Ministry of Social Affairs. Treatment exclusion criteria were (a) a current alcohol or drug problem, (b) a psychotic or personality disorder, (c) self-harming behavior, and (d) engagement in treatment elsewhere. Clients who met the exclusion criteria were referred either to specialized institutions or to voluntary help groups. Approval for the use of this data was obtained from the relevant ethical boards at the University of Southern Denmark (IRB approval: j.nr.2014-54-0853). Gender was recorded for $98.1 \%$ of the sample $(n=407$ women; $84.1 \%)$, age was recorded for $97.3 \%(M=36.4$ years, $S D=10.6)$, and years since the end of abuse was recorded for $59.3 \%(M=22.5$ years, $S D=11.8)$.

\section{Measures}

Traumatic experiences. The 30-item Harvard Trauma Questionnaire-Part III (HTQ; Mollica et al., 1992) was used to assess the occurrence of trauma-related symptoms, which respondents rate on a 4-point Likert scale $(1=$ not at all to $4=$ mostly); in the current study, only 20 items were used to assess the occurrence of DSM-5 PTSD symptoms in the past month (see Table 1). The Danish version of the HTQ has produced reliable and valid scores (Bach, 2003). Reported HTQ ratings according to the third edition (text revision) of the DSM (DSM-III-R) diagnostic criteria of PTSD have shown an $88.0 \%$ concordance with interview-based estimates of PTSD (Mollica et al., 1992).

Trauma symptoms. The Trauma Symptom Checklist (TSC; Briere \& Runtz, 1989) is a 33-item questionnaire used to assess general psychological distress. Respondents score answers on a 4 -point Likert scale $(1=$ never to $4=$ always $)$. The Danish version of the TSC has been used in a wide range of trauma populations with reports of good reliability and validity (Elklit, 1990). In the present study, only eight items were used to measure DSM-5 PTSD and ICD-11 Complex PTSD (see Table 1). 
Table 1

Items From the Harvard Trauma Questionnaire (HTQ) and Trauma Symptom Checklist (TSC) Mapped to the DSM-5 and ICD-11 Conceptualization of Posttraumatic Stress Disorder (PTSD) and Associated Response Frequencies for Danish Treatment-Seeking Adult Survivors of Childhood Sexual Abuse

\begin{tabular}{|c|c|c|c|c|c|c|c|c|c|c|}
\hline \multirow[b]{4}{*}{ Node Label } & \multirow[b]{4}{*}{ PTSD Experience } & \multirow{4}{*}{$\begin{array}{l}\text { Questionnaire } \\
\text { Item }\end{array}$} & \multicolumn{8}{|c|}{ Frequencies-Analytic Sample $^{d}$} \\
\hline & & & \multicolumn{8}{|c|}{$(n=473)$} \\
\hline & & & \multicolumn{2}{|c|}{ Not At All } & \multicolumn{2}{|c|}{ Rarely } & \multicolumn{2}{|c|}{ Sometimes } & \multicolumn{2}{|c|}{ Mostly } \\
\hline & & & $n$ & $\%$ & $n$ & $\%$ & $n$ & $\%$ & $n$ & $\%$ \\
\hline Thoughts ${ }^{\mathrm{a}, \mathrm{b}}$ & Recurrent thoughts & HTQ1 & 32 & 6.8 & 84 & 17.9 & 246 & 52.5 & 107 & 22.8 \\
\hline Nightmare $^{\mathrm{a}, \mathrm{b}, \mathrm{c}}$ & Recurrent nightmares & HTQ3 & 134 & 28.8 & 127 & 27.3 & 124 & 26.7 & 80 & 17.2 \\
\hline Flashback $^{\mathrm{a}, \mathrm{b}, \mathrm{c}}$ & Feel event happening again & HTQ2 & 124 & 26.4 & 146 & 31.1 & 161 & 34.3 & 39 & 8.3 \\
\hline $\begin{array}{l}\text { Physical }^{\mathrm{a}, \mathrm{b}}- \\
\text { Psychological }^{\mathrm{a}, \mathrm{b}}\end{array}$ & Sudden physical/psychological reaction & HTQ16 & 25 & 5.3 & 91 & 19.4 & 175 & 37.3 & 178 & 38.0 \\
\hline Avoid feelings ${ }^{\mathrm{a}, \mathrm{b}, \mathrm{c}}$ & Avoid thoughts or feelings of trauma & HTQ15 & 49 & 10.5 & 76 & 16.3 & 165 & 35.5 & 175 & 37.6 \\
\hline Avoid activities $^{\mathrm{a}, \mathrm{b}, \mathrm{c}}$ & Avoid activities that remind of trauma & HTQ11 & 85 & 18.5 & 88 & 19.1 & 102 & 22.2 & 185 & 40.2 \\
\hline Remember ${ }^{\mathrm{a}, \mathrm{b}}$ & Inability to remember parts of trauma & HTQ12 & 64 & 13.9 & 69 & 15.0 & 139 & 30.2 & 189 & 41.0 \\
\hline Future $^{\mathrm{a}, \mathrm{b}}$ & Feeling as if don’t have future & HTQ14 & 93 & 19.9 & 94 & 20.1 & 160 & 34.3 & 120 & 25.7 \\
\hline Blame $^{\mathrm{a}, \mathrm{b}}$ & Blame yourself for things that happened & HTQ19 & 135 & 29.1 & 91 & 19.6 & 137 & 29.5 & 101 & 21.8 \\
\hline Shame $e^{a, b, e}$ & Feel ashamed about things that happened & HTQ23 & 73 & 15.6 & 116 & 24.7 & 191 & 40.7 & 89 & 19.0 \\
\hline Guilt survived $^{\mathrm{a}, \mathrm{b}, \mathrm{e}}$ & Feel guilt for having survived & HTQ21 & 48 & 10.4 & 92 & 19.9 & 208 & 44.9 & 115 & 24.8 \\
\hline Guilt not doing ${ }^{\mathrm{a}, \mathrm{b}, \mathrm{e}}$ & Feel guilt not doing enough & HTQ31 & 90 & 19.4 & 88 & 18.9 & 145 & 31.2 & 142 & 30.5 \\
\hline Interest $^{\mathrm{a}, \mathrm{b}}$ & Less interest in daily activities & HTQ13 & 51 & 10.9 & 129 & 27.7 & 199 & 42.7 & 87 & 18.7 \\
\hline Detached $^{\mathrm{a}, \mathrm{b}}$ & Feel detached or withdrawn from people & HTQ4 & 48 & 10.3 & 73 & 15.6 & 190 & 40.7 & 156 & 33.4 \\
\hline Emotion $^{\mathrm{a}, \mathrm{b}}$ & Unable to show emotions & HTQ5 & 90 & 19.1 & 122 & 26.0 & 195 & 41.5 & 63 & 13.4 \\
\hline Irritable $^{\mathrm{a}, \mathrm{b}}$ & Feeling irritable or outbursts of anger & HTQ10 & 25 & 5.3 & 78 & 16.7 & 210 & 44.9 & 155 & 33.1 \\
\hline Reckless $^{\mathrm{a}, \mathrm{b}}$ & Want to harm yourself physically & $\mathrm{TSC} 21$ & 290 & 62.2 & 125 & 26.8 & 29 & 6.2 & 22 & 4.7 \\
\hline Guard $^{\mathrm{a}, \mathrm{b}, \mathrm{c}}$ & Feeing on guard & HTQ9 & 24 & 5.1 & 54 & 11.6 & 163 & 4.9 & 226 & 48.4 \\
\hline Jumpy $^{\mathrm{a}, \mathrm{b}, \mathrm{c}}$ & Feeling jumpy & HTQ6 & 51 & 10.9 & 106 & 22.6 & 135 & 28.8 & 177 & 37.7 \\
\hline Concentrate $^{\mathrm{a}, \mathrm{b}}$ & Difficulty concentrating & HTQ7 & 19 & 4.0 & 65 & 13.8 & 202 & 43.0 & 184 & 39.1 \\
\hline Sleep ${ }^{a, b}$ & Trouble sleeping & HTQ8 & 58 & 12.3 & 70 & 14.9 & 129 & 27.4 & 214 & 45.4 \\
\hline Depersonalization $^{\mathrm{b}}$ & Feel as if you are outside your body & $\mathrm{TSC} 32$ & 172 & 37.3 & 160 & 34.7 & 76 & 16.5 & 53 & 11.5 \\
\hline Derealization $^{\mathrm{b}}$ & A sense of unreality & TSC30 & 120 & 25.9 & 173 & 37.4 & 105 & 22.7 & 65 & 14.0 \\
\hline Temper ${ }^{\mathrm{c}}$ & Temper outburst you could not control & TSC16 & 143 & 30.4 & 195 & 41.5 & 67 & 14.3 & 65 & 13.8 \\
\hline Cry $^{\mathrm{c}}$ & Crying easily & TSC14 & 95 & 20.2 & 201 & 42.8 & 102 & 21.7 & 72 & 15.3 \\
\hline Isolated $^{c}$ & Feelings of inferiority or insecurity & TSC28 & 43 & 9.2 & 167 & 35.9 & 131 & 28.2 & 124 & 6.7 \\
\hline Blame $^{\mathrm{c}}$ & Blaming yourself & TSC29 & 53 & 11.5 & 142 & 30.7 & 147 & 31.8 & 120 & 26.0 \\
\hline Insecure $^{c}$ & Feeling isolated from others & TSC6 & 72 & 15.4 & 181 & 38.6 & 129 & 27.5 & 87 & 18.6 \\
\hline Rely on ${ }^{c}$ & Feeling that you have no one to rely upon & HTQ27 & 67 & 14.3 & 100 & 21.4 & 172 & 36.8 & 129 & 27.6 \\
\hline
\end{tabular}

Note. Minimal levels of missing data across response categories; valid percentage reported. DSM-5 = Diagnostic and Statistical Manual of Mental Disorders (5th ed.); ICD-11 = International Classification of Diseases (11th rev.).

${ }^{\mathrm{a}}$ Network $1=D S M-5$ PTSD. ${ }^{\mathrm{b}}$ Network $2=D S M-5$ PTSD with dissociative experiences. ${ }^{\mathrm{c}}$ Network $3=I C D-11$ Complex PTSD. ${ }^{\mathrm{d}}$ Corresponding response categories for TSC: never; yes, sometimes; yes, often; and yes, very often. ${ }^{\mathrm{e}} \mathrm{In} D S M-5$, these items are combined to reflect a single criterion, negative emotional state; for the purposes of this study, these items were modeled as separate experiences.

\section{Data Analysis}

Adults with complete missing data on all questionnaire items ( $n=11 ; 2.3 \%$ of the sample) were excluded. Three alternative PTSD networks were estimated in stages, using items from across the HTQ and TSC (see Table 1): (a) Network 1: DSM-5 (21 items), (b) Network 2: DSM-5 with dissociation (23 items, as per Hansen, Múllerová, Elklit, and Armour, 2016), and (c)
Network 3: ICD-11 CPTSD (12 items, as per Cloitre et al., 2013).

Stage 1: Network estimation and visualization. Edges (the association between two symptoms) were calculated by computing polychoric correlations between nodes (questionnaire items) using the state-of-the-art Pairwise Markov Random 
Field (PMRF; Epskamp, Borsboom, \& Fried, 2016; Epskamp \& Fried, 2016) for ordinal data, known as the Gaussian graphical model (GGM; Costantini et al., 2015; Lauritzen, 1996). Gaussian graphical model networks estimate a large number of parameters (e.g., 20 nodes require the estimation of 210 parameters: 20 threshold parameters and $20 \times 19 / 2=190$ pairwise association parameters) that likely result in some false-positive edges. Epskamp and colleagues (2016) directed that the least absolute shrinkage and selection operator (LASSO; Tibshirani, 1996) - a form of regularization which causes small connections to shrink to be exactly zero-be applied to construct a simple, parsimonious model. A well-established and fast algorithm for estimating LASSO regularization is the graphical LASSO (glasso; Friedman, Hastie, and Tibshirani, 2008), which is implemented in the $\mathrm{R}$ package qgraph (Epskamp, Cramer, Waldorp, Schmittmann, \& Borsboom, 2012; Friedman, Hastie, \& Tibshirani, 2014). Qgraph utilizes glasso in combination with the extended Bayesian information criterion (EBIC; Chen \& Chen, 2008) model selection to estimate a regularized GGM. The absence of an edge in this network indicates that two nodes are conditionally independent given all other nodes in the network (Costantini et al., 2015). For network visualization in gray scale, positive associations are full lines and negative associations are dashed. Thicker lines represent stronger connections and thinner lines represent weaker connections. Associations between PTSD indicators estimated in the networks are weighted but not directed, reflecting the magnitude of the association only. Qgraph implements the Fruchterman and Reingold (1991) algorithm, which positions strongly correlated nodes together.

Stage 2: Centrality estimation. Centrality, which reflects how connected a symptom is in a network, is indicated by three indices of node: strength, closeness, and betweenness (Opsahl, Agneessens, \& Skvoretz, 2010). Centrality indices are presented as standardized values. Items high in node strength, a measure of the sum of the weights of the edges (i.e., correlation magnitudes), are likely to exert strong direct influence over other nodes in the network. Closeness represents the average distance between a given node and the remaining nodes in the network; this may determine which PTSD symptoms are likely to be quickly affected by changes in other symptoms. The betweenness index reflects how important that node is for transmitting effects between other nodes in the network. Removal of items with high betweenness from a network increases the distance of other paths in the network (Costantini et al., 2015). High values on the centrality measures, which are presented graphically in qgraph, reflect a node's greater importance to the network.

Stage 3: Accuracy and stability estimation. We used the bootnet package in $\mathrm{R}$ to test the stability and accuracy of the networks (Epskamp et al., 2016; Fried \& Cramer, 2016). This analysis is necessary to determine the certainty with which the rank ordering of the edge weights and centrality indices can be interpreted. Specifically, we investigated the accuracy of the edge weights by constructing $95 \%$ confidence intervals (CI) around the edges and calculated the edge weights difference test that estimates whether edge weights differ from each other significantly. Further, we estimated the stability of the order of the estimation by subsetting bootstrap (i.e., dropping participants and reestimating the network); if the order of the centrality estimates obtained from a network with substantially fewer participants is highly correlated to those obtained from a network analysis of all participants, the centrality estimates are viewed as stable. The centrality stability (CS) coefficient should be at a minimum 0.25 , but preferably 0.5 or higher (Epskamp et al., 2016).

\section{Results}

\section{Preliminary Analyses}

The distributions of HTQ and TSC items satisfied skewness criteria for normality. As presented in Table 1, experiences of arousal, such as hypervigilance, occurred frequently, whereas experiences related to self-destructive behavior occurred less so.

\section{Network 1: DSM-5}

Visual inspection (Figure 1, Panel A) revealed several strong positive correlations between thoughts, flashbacks, and physical-psychological reactions as well as between these items and experiences of shame and avoid activities. Guilt survived and future were strongly associated with each other as were guilt not doing and blame, but these items were not associated with other experiences in the NACM cluster. Weaker associations emerged between guard and jumpy and the items sleep and concentrate. Remember was positively associated with emotions. The results of the accuracy and stability testing for this network (see Supplementary Materials) indicated substantial interrelatedness, with correlations of .75 between node strength and closeness, .73 between node strength and betweenness, and .80 between closeness and betweenness. Moreover, CS coefficients for node strength, closeness, and betweenness were $.51, .36$, and .21 , respectively. Combined, these findings justified a focus on the stable estimates of node strength and closeness in the main report, with the findings related to betweenness relegated to the Supplementary Material. Figure 2 (Panel A) presents standardized centrality estimates; the five nodes with the highest centrality indices were flashbacks, future, guilt survived, detached, and nightmares. Nightmares (Node 2) had a strong strength index but also the highest closeness estimate across all experiences. Irritable and remember produced the lowest estimates across both centrality indices. 


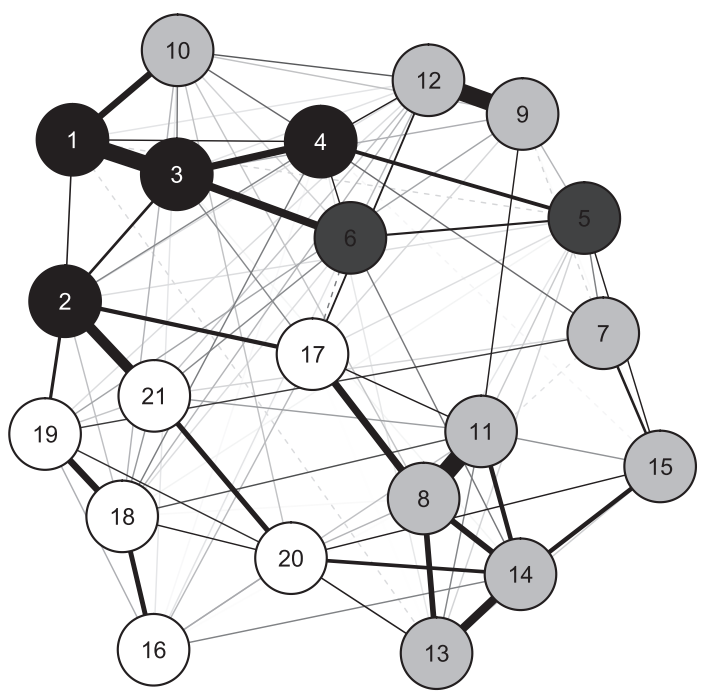

PTSD_Intrusions

- 1: Thoughts

- 2: Nightmar

- 3: Flash

PTSD_Avoidance

- 5: Avoid feelings

6: Avoid activities

PTSD_NACM

- 7: Rememb

8: Future

- 9: Blame

- 11: Guilt survive

- 12: Guilt not doing

- 13: Less interest

- 14: Detached

15: Emotion

PTSD_Arousal

16: Irritable

17: Reckless

18: Guard

19: Jumpy
20: Concentrate

21: Sleep
o
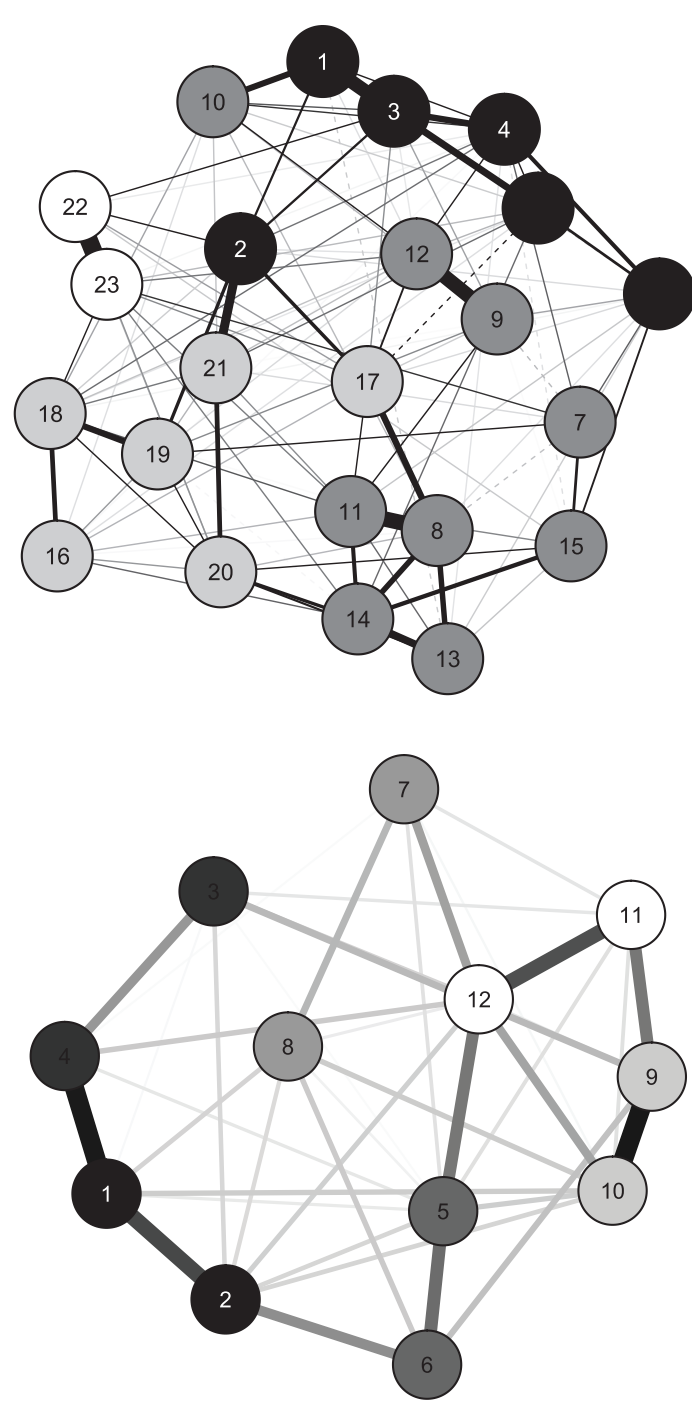

Panel A

PTSD_reexperience - 1: Thoughts - 3: Flash

- 4: Physical_psychological

PTSD_Avoidance

- 5: Avoid feelings

- 6: Avoid activities

PTSD_NACM

- 7: Remember

- 8: Future

- 9: Blame

- 10: Shame

- 11: Guilt survived

- 13: Less interest

- 14: Detached

- 15: Emotion

PTSD_Arousal

- 16: Irritable

17: Reckless

- 18: Guard

- 19: Jumpy

20: Concentrate

- 21: Sleep

Dissociation

22: Depersonalisation

- 23: Derealization

\section{Panel B}

PTSD_reexperience

- 1: Flash

1: Flash
2: Nightmare

PTSD_Avoidanc

3: Avoid feelings

- 3: Avoid feelings

PTSD_Sense_Threat

- 5: Guard

- 5: Guard

CPTSD_affect_regulation

- 7: Temper

- 7: Temper
- 8: Cry

CPTSD_negative_selfconcept

9: Insecure

$\circ$ 9: Insecure
o 10: Blame

CPTSD interpersonal

11: Isolated

12: Rely on

Figure 1. Gaussian graphical model (GGM) networks of Harvard Trauma Questionnaire and Trauma Symptom Checklist items depicting a Diagnostic and Statistical Manual of Mental Disorders (5th ed.; DSM-5 [Panel A]), DSM-5 with dissociation (Panel B), and International Classification of Diseases (11th rev.) complex posttraumatic stress disorder (CPTSD [Panel C]) experiences among adult survivors of childhood sexual abuse $(n=473)$. PTSD $=$ posttraumatic stress disorder; $\mathrm{NACM}=$ negative alterations in cognition and mood. 

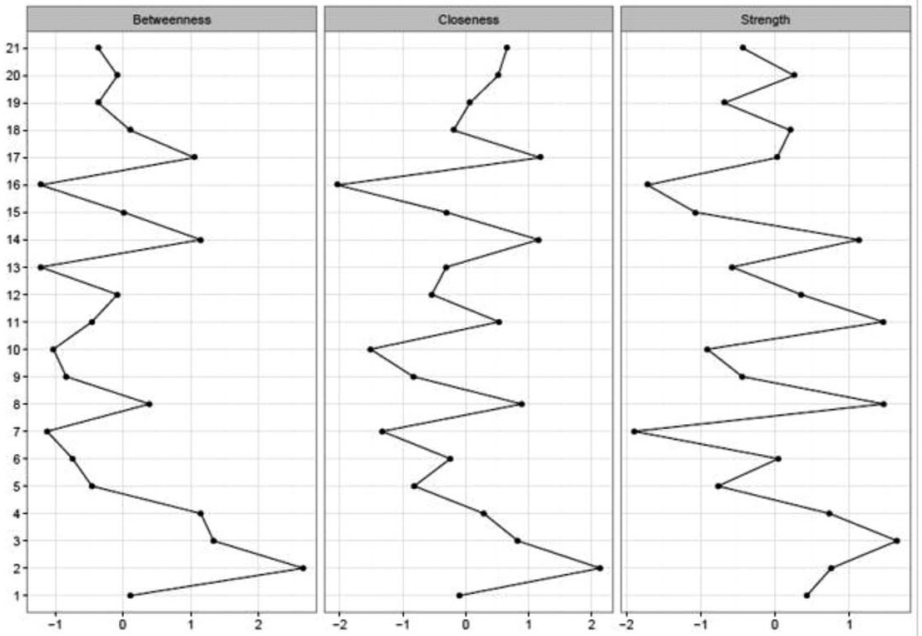

PTSD_Intrusions

1: Thoughts

2: Nightma

- 4: Physical_psychological

PTSD_Avoidance

- 5: Avoid feelings

- 6: Avoid activities

PTSD_NACM

- 7: Remember

- 9: Blame

- 10: Bame

- 11: Guilt survive

- 12: Guilt not doing

- 13: Less interest

- 14: Detached

- 15: Emotion

PTSD_Arousa

- 16: Irritable

- 17: Reckless

- 18: Guard

- 19: Jumpy

- 20: Concentrate

- 21: Sleep
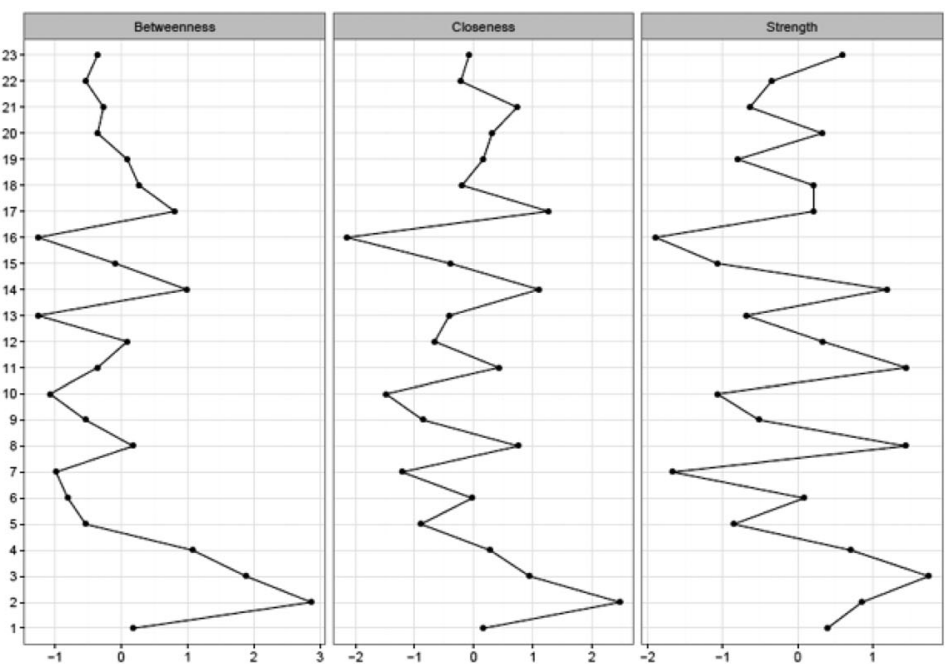

PTSD_reexperience

1: Thoughts

: 2: Nightm

3: Flash

4: Physical_psychological

PTSD_Avoidance

- 5: Avold feelings

PTSD NACM

- 7: Remember

- 8: Future

- 9: Blame

- 10: Shame

- 11: Guilt survived

12: Guilt not doing

-13: Less interes

- 14: Detached
- 15: Emotion

PTSD_Arousal

- 16: Irritable

17: Reckless

18: Guard

19: Jumpy

20: Concentrate

21: Sleep

Dissociation

22: Depersonalisation

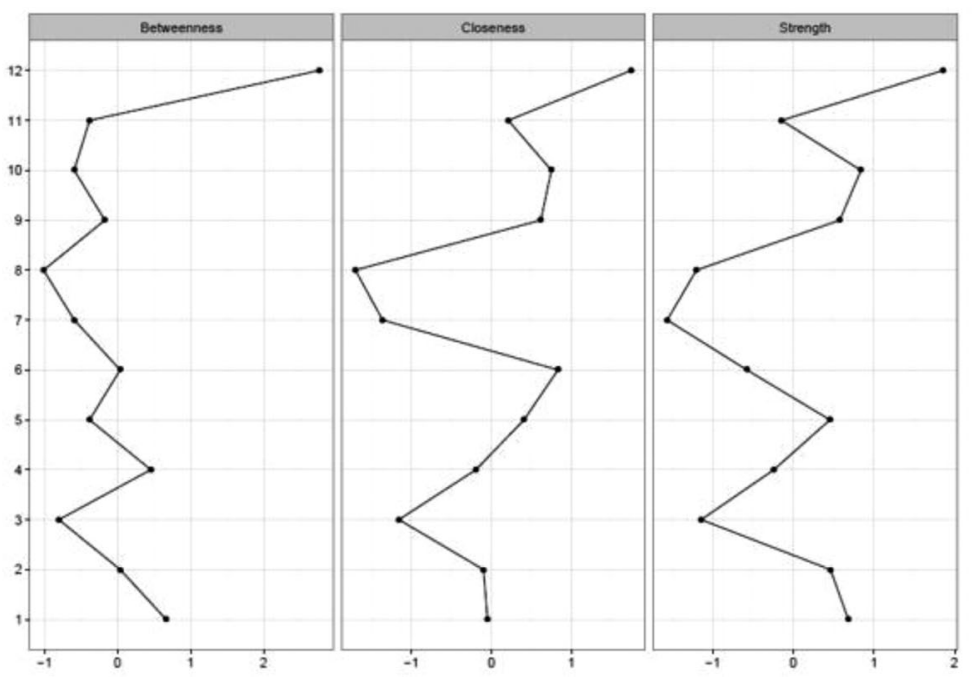

PTSD reexperience

- 1: Flash

- 2: Nightmare

PTSD_Avoidance

-3: Avoid feelings

- 4: Avoid activities

PTSD_Sense_Threat

- 5: Guard

- 6: Jumpy

CPTSD_affect_regulation

\section{Panel C}

- 7: Temper

- 8: Cry

CPTSD_negative_selfconcept

- 9: Insecure

- 10: Blame

CPTSD_interpersonal

- 11: Isolated

- 12: Rely on

Figure 2. Centrality estimates from Gaussian graphical model (GGM) network of Harvard Trauma Questionnaire and Trauma Symptom Checklist items depicting a Diagnostic and Statistical Manual of Mental Disorders (5th ed.; DSM-5 [Panel A]), DSM-5 with dissociation (Panel B), and International Classification of Diseases (11th rev.) complex posttraumatic stress disorder (CPTSD [Panel C]) experiences among adult survivors of childhood sexual abuse $(n=473)$. PTSD $=$ posttraumatic stress disorder; $\mathrm{NACM}=$ negative alterations in cognition and mood. 


\section{Network 2: DSM-5 (With Dissociation)}

The strongest positive associations emerged between depersonalization and derealization, with weaker associations evident between these items and other experiences (Figure 1, Panel B). Depersonalization was associated mainly with the experiences nightmares and flashbacks, whereas derealization was mainly associated with experiences in the arousal and NACM clusters (e.g., concentrate and remember). As outlined in the Supplementary Materials, the CS coefficients for node strength, closeness, and betweenness were $.52, .28$, and .21 , respectively. The rank ordering of the strongest edges changed from Network 1 to Network 2; specifically, the edge weight between derealization and depersonlization was ranked first, followed by future and guilt survived (second; first in Network 1), blame and guilt not doing (third; second in Network 1), and thoughts and flashbacks (fourth; third in Network 1). Of the pair, depersonalization had the strongest strength index but was weaker than the top five nodes in Network 1 (Figure 2, Panel B).

\section{Network 3: ICD-11 CPTSD}

The strongest associations emerged between rely on and isolated as well as insecure and blame; the association between temper and cry was weaker (Figure 1, Panel C). The CS coefficients for node strength, closeness, and betweenness were $.59, .28$, and .13, respectively (see Supplementary Materials). Nodes that reflected affect regulation had the lowest strength estimates, which raised concerns about their importance in the network. Alternatively, rely on was the most important experience in this network (Figure 2, Panel C).

\section{Discussion}

The findings from this study, which sought to understand and explain the core experiences of adult survivors of CSA trauma from a network perspective, can be summarized succinctly. Strong associations between the three pairs of experiences emerged: future and guilt survived, blame and guilt not doing, and thoughts and flashbacks. When depersonalization and derealization were included in the network, the strength of this association outranked the three aforementioned associations. Depersonalization in particular was an important element of the posttrauma experience. In the CPTSD network, interpersonal problems and negative self-concept (i.e., DSO) seemed to be core elements of the posttrauma experience, whereas difficulties regulating mood were less so. Collectively, these findings suggest support for the study's hypotheses. Before a detailed discussion of the findings, some study limitations are worth mentioning. Survivors were predominantly female, educated, married, in middle adulthood, and free from substance use problems and other mental health difficulties; these characteristics preclude generalizability of this study's findings to other trauma groups. Experiences of PTSD were assessed at one time point in adulthood, which was, for most individuals, many years after the trauma had ended. Although there are ongoing efforts to develop a measure of ICD-11 CPTSD (e.g., the International Trauma Questionnaire; Hyland et al., 2017), this study was limited to approximating CPTSD using items from other measures. Given the cross-sectional study design, we were unable to determine whether the most central experiences activated other experiences or were activated by other experiences, or whether a reciprocal association was the most plausible explanation. Finally, the sample size could be perceived as merely adequate for estimating a network with 23 nodes.

The findings of this paper, which relate to a highly specific trauma survivor group, indicated that, many years following traumatic CSA, experiences relating to NACM appear more central to the posttrauma experience than experiences of arousal. If replicated elsewhere, this may have an important clinical implication: In the immediate to short-term aftermath of a trauma, clinical interventions that focus on reducing physiological and psychological arousal may be most effective, whereas in the long-term, clinical interventions that focus on modifying dysfunctional cognitive and/or emotional factors may be required. It was interesting to note that the two dissociation experiences were highly associated with one another but relatively isolated from all other PTSD symptoms. On one hand, this finding could be interpreted as an indication of support for the notion of a PTSD subtype (Armour, Karstoft, \& Richardson, 2014; Lanius et al., 2014). On the other hand, it may indicate that these symptoms have little in common with the other PTSD symptoms and therefore their inclusion within a PTSD profile could be questioned (Cloitre et al., 2013; Maercker et al., 2013).

Finally, we focused our attention on the suitability of network analysis to identify the PTSD experiences of Danish adults. Despite a growing number of studies that have used this modeling framework (Afzali et al., 2017; Bryant et al., 2017), critics contest that the methodology is in its infancy and there should be legitimate concerns about the approach (Ashton \& Lee, 2012). In response, we note that the findings of the current (and past) network models suggest a considerable degree of similarity to traditional latent variable models of PTSD. For example, in each of the current networks, the PTSD symptoms cluster in a manner that largely reflects the four-factor model in the DSM-5 (American Psychiatric Association, 2013), the dissociative subtype, and the six-factor model of ICD-11 (World Health Organization, 2018) CPTSD. The network approach offers no obvious explanation for why the intrusions, avoidance, NACM, arousal, and dissociative symptoms are intra- and interrelated in the manner in which they are. Indeed, if latent variable models were incorrect and PTSD symptoms were simply related to one another in a causal network with no underlying latent structure, it would seem improbable that these symptoms should cluster together in a consistent manner across multiple studies and much less so in a manner similar to what has been evidenced in the latent variable modeling literature. Of course, the current results indicate that the symptoms are not clustering in a 
manner that perfectly reflects the four-factor model of DSM-5 PTSD (e.g., Items 9, 10, and 12 are disconnected from the rest of the NACM symptoms, and some of the arousal symptoms are unconnected to one another; APA 2013). The current, and prior, findings could be used as a potential exploratory approach to identify symptom covariation patterns that could aid in the development of new factorial models of psychiatric disorders. Furthermore, the network approach seems useful in terms of identifying "core symptoms" of a given disorder and thus useful for item and/or symptom reduction purposes. Consider, for example, two arousal items in this study that were strongly connected (guard and jumpy) but largely unrelated to the other arousal symptoms. These two items were selected by the ICD11 Working Group for Trauma and Stress-Related Disorders as the core hyperarousal symptoms to be included within the recently published $I C D-11$ model of PTSD (Karatzias et al., 2017). The results of the current study, and those of McNally et al. (2014), support the notion that these two items are distinct measures of hyperarousal. Thus, rather than viewing network analysis and latent variable models as competing methodologies for understanding psychopathology, it may be possible instead to consider both as complimentary techniques that achieve and reveal more together than they do separately.

\section{References}

Afzali, M. H., Sunderland, M., Teesson, M., Carragher, N., Mills, K., \& Slade, T. (2017). A network approach to the comorbidity between posttraumatic stress disorder and major depressive disorder: The role of overlapping symptoms. Journal of Affective Disorders, 208, 490-496. https://doi.org/10.1016/j.jad.2016.10.037

American Psychiatric Association. Diagnostic and statistical manual of mental disorders (5th ed.). Washington, DC: Author.

Armour, C., Fried, E. I., Deserno, M. K., Tsai, J., \& Pietrzak, R. H. (2017) A network analysis of $D S M-5$ posttraumatic stress disorder symptoms and correlates in US military veterans. Journal of Anxiety Disorders, 45, 49-59.

Armour, C., Karstoft, K.-I., \& Richardson, J. D. (2014). The co-occurrence of PTSD and dissociation: Differentiating severe PTSD from dissociativePTSD. Social Psychiatry and Psychiatric Epidemiology, 49, 1297-1306.

Ashton, M. C., \& Lee, K. (2012). On models of personality structure. European Journal of Personality, 26, 433-434.

Bach, M. E. (2003). En empirisk belysning og analyse af "Emotional Numbing" som eventuel selvstændig faktor i PTSD [An empirical investigation and analysis of "Emotional Numbing" as a possible independent factor in PTSD]. Psykologisk Tidsskriftserie, 5, 1-199.

Borsboom, D. (2008). Latent variable theory. Measurement, 6, 25-53.

Borsboom, D., Cramer, A. O., Schmittmann, V. D., Epskamp, S., \& Waldorp, L. J. (2011). The small world of psychopathology. PloS one, 6(11), e27407.

Briere, J., \& Runtz, M. (1989). The Trauma Symptom Checklist (TSC-33) early data on a new scale. Journal of Interpersonal Violence, 4, 151-163.

Bryant, R. A., Creamer, M., O'Donnell, M., Forbes, D., McFarlane, A. C., Silove, D., \& Hadzi-Pavlovic, D. (2017). Acute and chronic posttraumatic stress symptoms in the emergence of posttraumatic stress disorder: A network analysis. JAMA psychiatry, 74, 135-142. https://doi.org/10.1001/jamapsychiatry.2016.3470
Chen, J., \& Chen, Z. (2008). Extended Bayesian information criteria for model selection with large model spaces. Biometrika, 95, 759-771.

Clark, D. A., \& Beck, A. T. (2011). Cognitive therapy of anxiety disorders: Science and practice: New York, NY: Guilford Press.

Cloitre, M., Garvert, D. W., Brewin, C. R., Bryant, R. A., \& Maercker, A. (2013). Evidence for proposed ICD-11 PTSD and complex PTSD: A latent profile analysis. European Journal of Psychotraumatology, 4. https://doi.org/10.3402/ejpt.v4i0.20706

Costantini, G., Epskamp, S., Borsboom, D., Perugini, M., Mõttus, R., Waldorp, L. J., \& Cramer, A. O. (2015). State of the aRt personality research: A tutorial on network analysis of personality data in R. Journal of Research in Personality, 54, 13-29.

Cramer, A. O., Sluis, S., Noordhof, A., Wichers, M., Geschwind, N., Aggen, S. H., ... Borsboom, D. (2012). Dimensions of normal personality as networks in search of equilibrium: You can't like parties if you don't like people. European Journal of Personality, 26, 414-431.

Elklit, A. (1990). Måling af belastninger efter voldeligt overfald med TSC-33 traume symptom checkliste [Measuring distress after violent assault with TSC-33]. Nordisk Psykologi, 42, 281-289.

Elklit, A., Christiansen, D., Palic, S., Karsberg, S., \& Eriksen, S. (2014). Impact of traumatic events on posttraumatic stress disorder among Danish survivors of sexual abuse in childhood. Journal of Child Sexual Abuse, 23, 918-934.

Epskamp, S., Borsboom, D., \& Fried, E. I. (2016). Estimating psychological networks and their accuracy: A tutorial paper. ArXiv Preprint, 501, 1-25.

Epskamp, S., Cramer, A. O., Waldorp, L. J., Schmittmann, V. D., \& Borsboom, D. (2012). Qgraph: Network visualizations of relationships in psychometric data. Journal of Statistical Software, 48, 1-18.

Epskamp, S., \& Fried, E. I. (2016). A primer on estimating regularized psychological networks. arXiv preprint arXiv:1607.01367.

Fried, E. I., \& Cramer, A. O. (2016). Moving forward: Challenges and directions for psychopathological network theory and methodology. Perspectives on Psychological Science, 12, 999-1020. https://doi. org/10.1177/1745691617705892

Fried, E. I., Eidhof, M. B., Palic, S., Costantini, G., Huisman-van Dijk, H. M., Bockting, C. L., ... Karstoft, K. -I. (2018). Replicability and generalizability of posttraumatic stress disorder (PTSD) networks: A crosscultural multisite study of PTSD symptoms in four trauma patient samples. Clinical Psychological Science, 6, 335-351. https://doi.org/10.1177/ 2167702617745092

Friedman, J., Hastie, T., \& Tibshirani, R. (2008). Sparse inverse covariance estimation with the graphical lasso. Biostatistics, 9, 432-441.

Friedman, J., Hastie, T., \& Tibshirani, R. (2014). glasso: Graphical lassoestimation of Gaussian graphical models. R package version 1.8. Available from https://cran.r-project.org/web/packages/glasso/glasso.pdf

Fruchterman, T. M., \& Reingold, E. M. (1991). Graph drawing by forcedirected placement. Software: Practice and Experience, 21, 1129-1164.

Hansen, M., Múllerová, J., Elklit, A., \& Armour, C. (2016). Can the dissociative PTSD subtype be identified across two distinct trauma samples meeting caseness for PTSD? Social Psychiatry and Psychiatric Epidemiology, 51, 1159-1169. https://doi.org/10.1007/s00127-016-1235-2

Hyland, P., Shevlin, M., Brewin, C., Cloitre, M., Downes, A., Jumbe, S., .. Roberts, N. (2017). Validation of post-traumatic stress disorder (PTSD) and complex PTSD using the International Trauma Questionnaire. Acta Psychiatrica Scandinavica, 136, 313-322.

Karatzias, T., Shevlin, M., Fyvie, C., Hyland, P., Efthymiadou, E., Wilson, D., ... Cloitre, M. (2017). Evidence of distinct profiles of posttraumatic stress 
disorder (PTSD) and complex posttraumatic stress disorder (CPTSD) based on the new ICD-11 Trauma Questionnaire (ICD-TQ). Journal of Affective Disorders, 207, 181-187.

Kendler, K. S. (2005). "A gene for ...": the nature of gene action in psychiatric disorders. American Journal of Psychiatry, 162, 1243-1252.

Kubany, E. S., \& Manke, F. P. (1995). Cognitive therapy for trauma-related guilt: Conceptual bases and treatment outlines. Cognitive and Behavioral Practice, 2, 27-61.

Lanius, R., Wolf, E., Miller, M., Frewen, P., Vermetten, E., Brand, B., .. Resick, P. (2014). The dissociative subtype of PTSD. In M. Friedman, T. Keane, \& P. Resick (Eds.), Handbook of PTSD: Science and practice (2nd ed.; pp. 234-250). New York, NY: Guilford Press.

Lauritzen, S. L. (1996). Graphical models. Oxford, UK: Clarendon Press.

Maercker, A., Brewin, C. R., Bryant, R. A., Cloitre, M., Reed, G. M., van Ommeren, M., ... Llosa, A. E. (2013). Proposals for mental disorders specifically associated with stress in the International Classification of Diseases-11. The Lancet, 381, 1683-1685.

McNally, R. J., Robinaugh, D. J., Wu, G. W., Wang, L., Deserno, M. K., \& Borsboom, D. (2014). Mental disorders as causal systems: A network approach to posttraumatic stress disorder. Clinical Psychological Science, 3, 836-849. https://doi.org/10.1177/2167702614553230

Mollica, R. F., Caspi-Yavin, Y., Bollini, P., Truong, T., Tor, S., \& Lavelle, J. (1992). The Harvard Trauma Questionnaire: Validating a cross-cultural instrument for measuring torture, trauma, and posttraumatic stress disorder in Indochinese refugees. The Journal of Nervous and Mental Disease, 180, 111-116.
O’Donnell, M. L., Elliott, P., Lau, W., \& Creamer, M. (2007). PTSD symptom trajectories: From early to chronic response. Behaviour Research andTtherapy, 45, 601-606.

Opsahl, T., Agneessens, F., \& Skvoretz, J. (2010). Node centrality in weighted networks: Generalizing degree and shortest paths. Social Networks, 32, 245 251.

Roberts, A. L., Gilman, S. E., Breslau, J., Breslau, N., \& Koenen, K. C. (2011). Race/ethnic differences in exposure to traumatic events, development of posttraumatic stress disorder, and treatment-seeking for posttraumatic stress disorder in the United States. Psychological Medicine, $41,71-83$.

Robinaugh, D. J., LeBlanc, N. J., Vuletich, H. A., \& McNally, R. J. (2014). Network analysis of persistent complex bereavement disorder in conjugally bereaved adults. Journal of Abnormal Psychology, 123, 510.

Schell, T. L., Marshall, G. N., \& Jaycox, L. H. (2004). All symptoms are not created equal: The prominent role of hyperarousal in the natural course of posttraumatic psychological distress. Journal of Abnormal Psychology, 113, 189.

Sullivan, C. P., Smith, A. J., Lewis, M., \& Jones, R. T. (2016). Network analysis of PTSD symptoms following mass violence. Psychological Trauma: Theory, Research, Practice, and Policy, 10, 58-66. https://doi.org/10.1037/tra0000237

Tibshirani, R. (1996). Regression shrinkage and selection via the lasso. Journal of the Royal Statistical Society. Series B (Methodological), 58, 267-288.

World Health Organization. International Classification of Diseases (11th rev.). Geneva, Switzerland: Author. 